\title{
THE INFLUENCE OF THE TRACK AXIS CURVATURE AT RAILWAY FILLER-BEAM DECK BRIDGES
}

\begin{abstract}
RĂZVAN MARIAN STĂNESCU - Assistant Professor, Technical University of Civil Engineering, Faculty of
\end{abstract} Railways, Roads and Bridges, e-mail: razvan@cfdp.utcb.ro

\begin{abstract}
The article presents a comparative study between the simplified method calculation proposed by the prescriptions of design codes and the analysis with the FEM program LUSAS [1], regarding the influence of the curvature of the track axis at railway bridges with steel beams embedded in concrete.

The study was made on three simply supported bridges with the openings chosen so as to cover the openings used for this constructive solution, namely $10 \mathrm{~m} \leq \mathrm{L} \leq 30 \mathrm{~m}$. For each analysed opening the curve radius of the track axis was varied,in the domain in which are representative as effects, namely $100 \mathrm{~m} \leq R \leq 1500 \mathrm{~m}$. In the case of Lusas FEM analysis, a physically nonlinear analysis it was previously carried out, after which the cracked concrete was removed, as its participation in the structural stiffness is practically non-existent.

Studying the outcomes revealed by the two calculations presented, it can be concluded that the simplified method proposed by the design codes leads to a overvaluation of the track axis curvature influence at the railway filler-beam deck bridges.
\end{abstract}

Keywords: simplified method, 3D modelling, nonlinear analysis, multi-cracked concrete

\section{Introduction}

In all the existing design codes of railway filler-beam deck bridges, as well as in the technical literature, there is little information about the influence of the curvature of the track axis, with respect to the design and calculation of these types of structures.

Until the development and introduction of the European standards, the design of railway fillerbeam deck bridges was made according to the recommendations of the UIC Code 773-4 R [2], attend by pre-dimensioning tables for these types of structure, which were based primarily on research conducted by the Committee ORE D-123 of the International Union of Railways. According to UIC 773-4 R [25], the calculation is made on a singular longitudinal beam, with steel-concrete composite cross-section.

Regarding the influence of the track axis curvature, the current practice of design uses the simplified method of the Romanian standard SR 98-1911 [3], which is based on Engesser's theory, developed since the late 19th century.

Furthermore, the European standards don't particulary bring into light those design situations. As for the design situations in which the cross load distribuition is not symmetrical, the European standards recommend the analysis with the FEM programs.

The purpose of this article is to analyse the influence of the track axis curvature by using a program based on FEM, as well as compare it with the outcome obtained through the simplified method.

\section{Presentation of the case studies}

The Design Tables for Filler Beam Railway Bridges [4], published by the International Union of Railways, has been the guideline for the constructive solutions of the three analysed bridges:

- Bridge with $L=10 m$ opening

- Bridge with $L=20 m$ opening 
- Bridge with $L=30 m$ opening

For the bridge with the $L=10 \mathrm{~m}$ opening and a B track category, at a maximum speed of $160 \mathrm{Km} / \mathrm{h}$, the constructive solution consists of six steel beams HEA 400 in cross-section, as shown in Figure 1. For the bridge with the $L=20 \mathrm{~m}$ opening the constructive solution consists of six HEB 800 steel beams and for the bridge with the $L=30 \mathrm{~m}$ opening the constructive solution consists of six HLB 1100 steel beams.

In all three cases a deck width of $4 \mathrm{~m}$ was obtained.
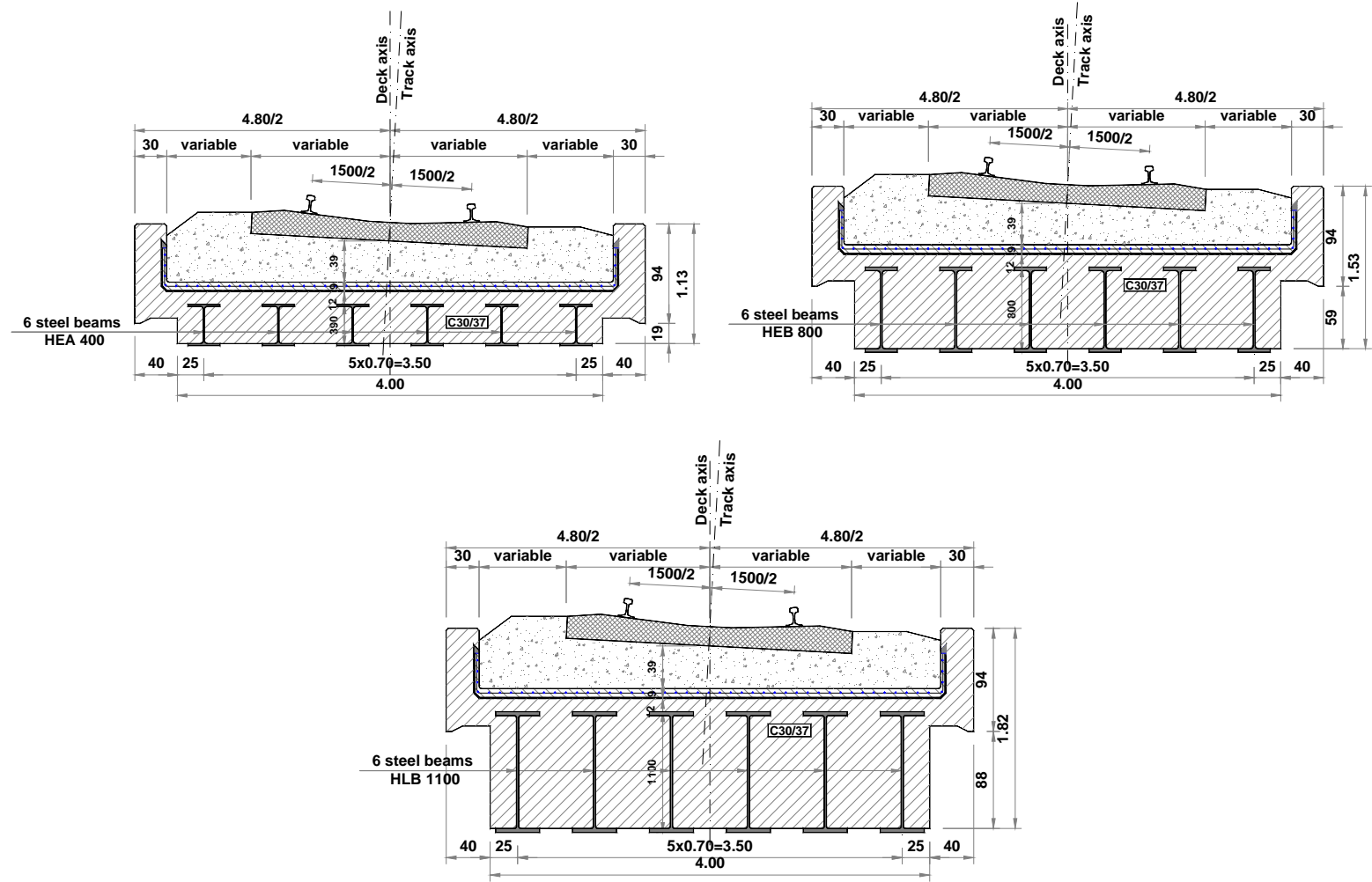

Fig. 1 - Constructive solutions for the 3 simply supported bridges analysed

\section{The simplified method}

By applying this method, the calculation is reduced to that of bridges in alignment. The various direct or indirect effects that arise at bridges with the track axis in curve are taken into account by introducing 3 equivalent eccentricities. These eccentricities take into consideration the curve radius, the cant track and the track axis misalignment up against the longitudinal axis of the structure.

$e_{s}$ - eccentricity due to the cant track;

$e_{c}$ - eccentricity due to the centrifugal force;

$e_{n}$ - eccentricity due to the track axis misalignment up against the longitudinal axis of the structure.

Please note that the cant track has a positive effect and thus, the corresponding eccentricity is subtracted from the calculation of the total eccentricity.

$$
e_{t o t}=e_{c}+e_{n}-e_{s}
$$

The calculation of the equivalent eccentricities for the section at the mid-span of the bridge was made according to the Romanian standard SR 1911-98 [3]. The values of the equivalent eccentricities are shown in the graph on Figure 2. 


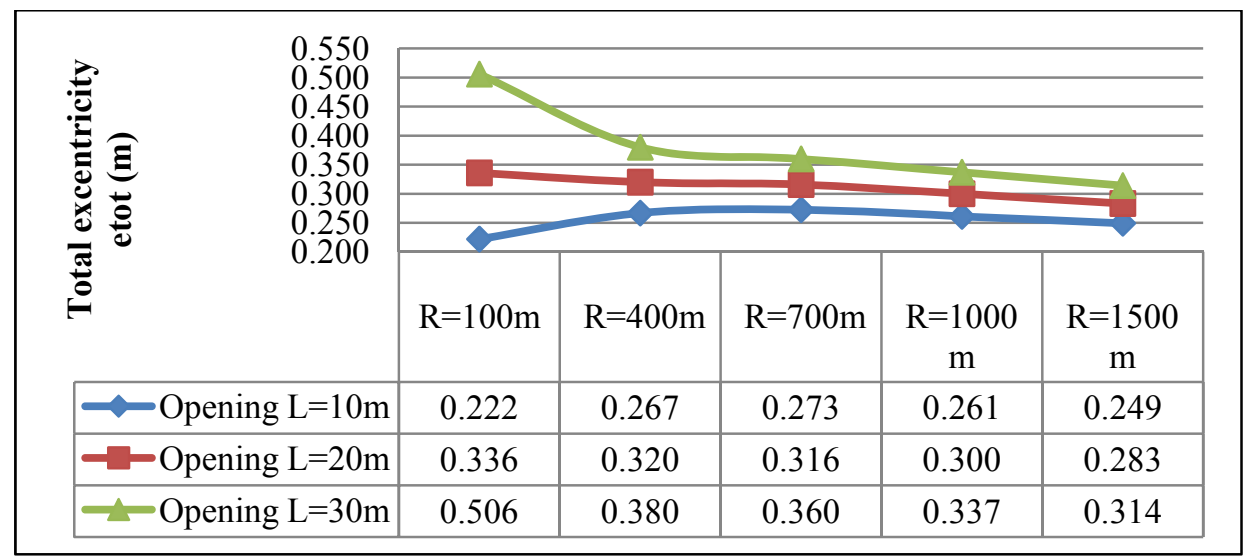

Fig. 2 - The variation of total eccentricity with the opening and the curve radius

The calculation was done considering bridge deck divided into longitudinal beams. A longitudinal beam is composed by steel beam and the associated embedded concrete, as shown in Figure 3.

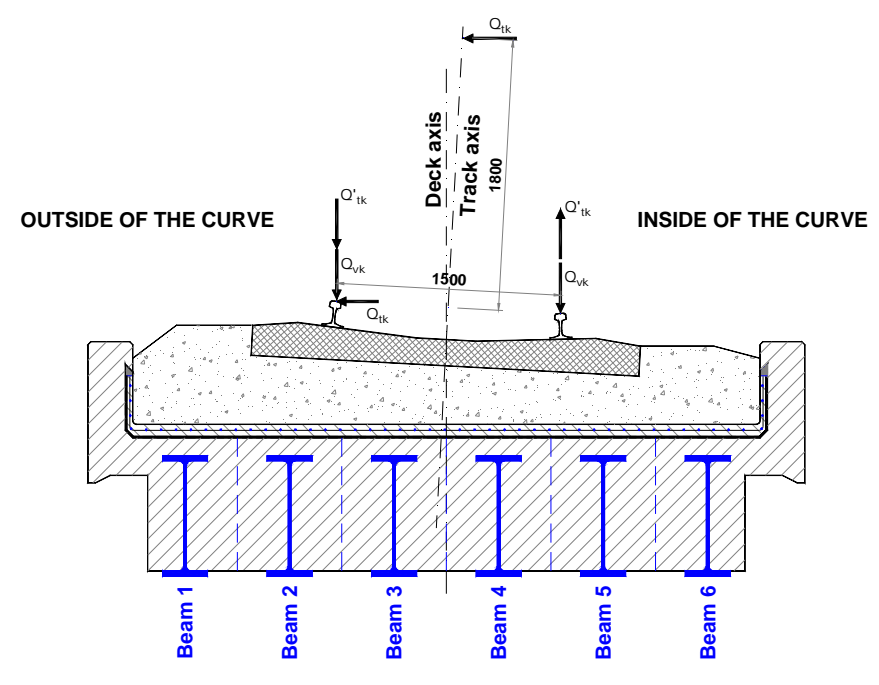

Fig. 3 - Notation of the longitudinal beams in the cross-section

The bending moments in the section at the mid-span of the bridge, for each longitudinal beam of the bridge deck, were acquired by multiplying the total bending moment of the deck with the repartition coefficients of the transversal loads, which are exemplified for a certain eccentricity in Figure 4.

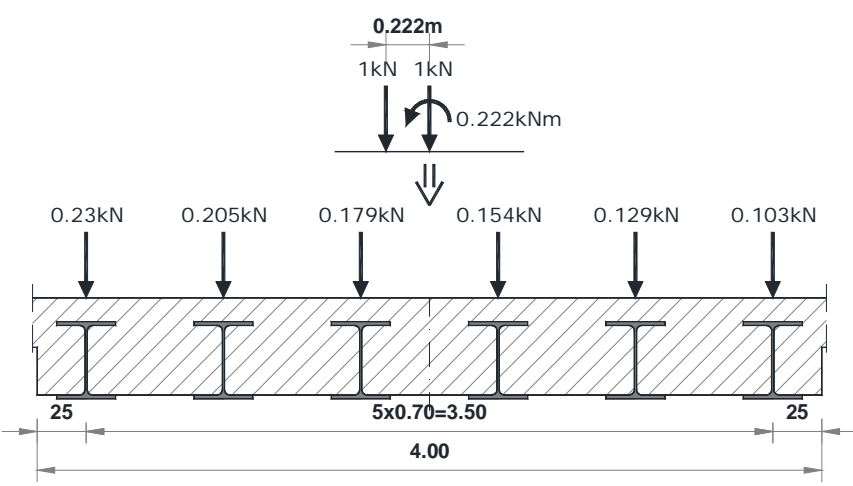

Fig. 4 - The calculation principle of the repartition coefficients of the transversal loads

The repartition coefficients of the transversal loads and the bending moments on each beam are presented in Table 1. 
The repartition coefficients of the transversal loads and the bending moments

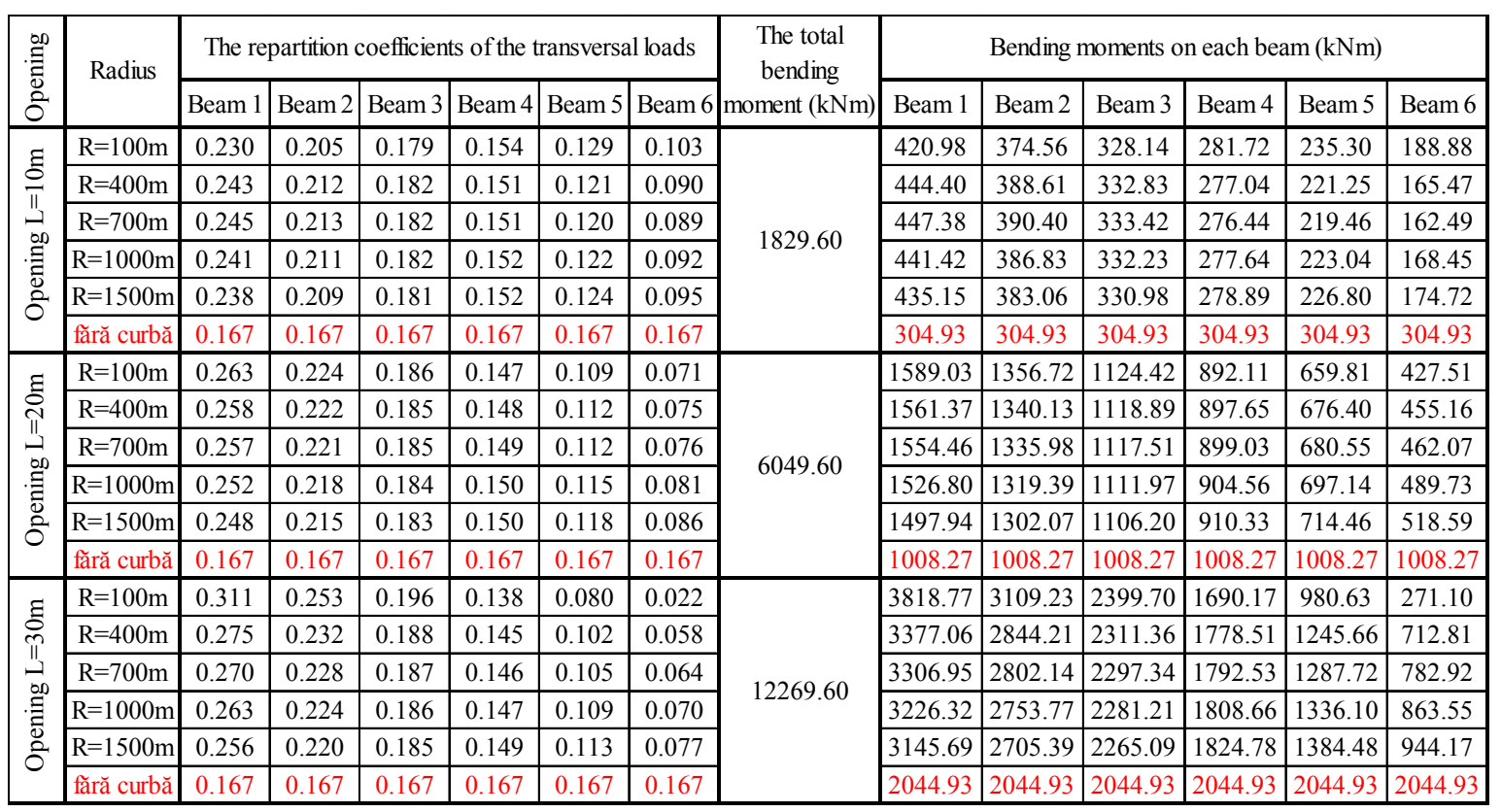

\section{The analysis with FEM program Lusas [1]}

\subsection{Physically nonlinear analysis of a longitudinal beam}

It was previously carried out a physically nonlinear analysis on a longitudinal beam of the crosssection, meshed with 3D finite elements, aiming the developments of the cracks produced by the permanent weight and the traffic actions. The analysis was performed for each of the three chosen openings. To decrease the calculation volume, the beam was analyzed by symmetry.

\subsubsection{Bridge with $L=10 m$ opening}

\section{Loading with permanent weight}

The calculation model contains 3400 "3D” finite elements type "Stress" HX8M and 4386 nodes, resulting in 13158 degrees of freedom, as shown in Figure 5.

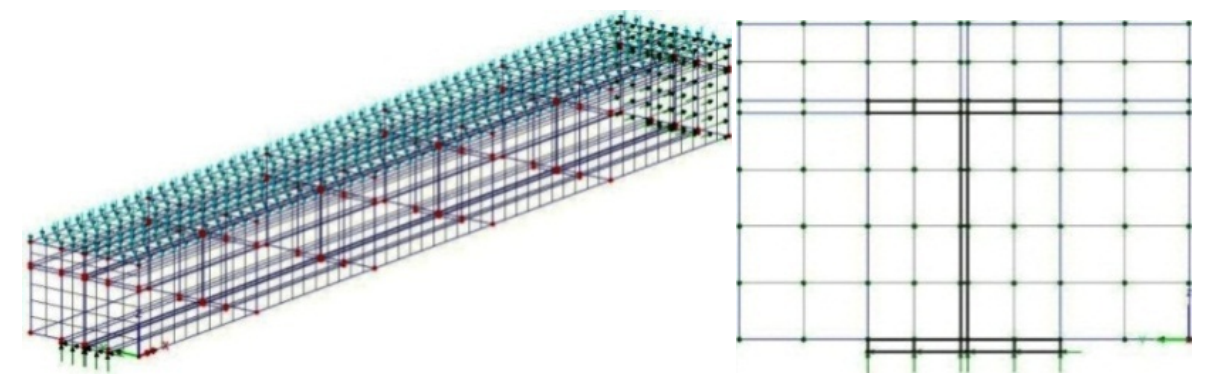

Fig. 5 - The calculation model and the cross-section of the beam for permanent weight

The two materials that make up the cross-section were chosen with nonlinear behavior. For steel, a material with elastoplastic behavior, type Stress Potential was chosen (based on the Von Mises's theory). For concrete, also a material with elastoplastic behavior was chosen. This one is specific in the Lusas FEM program [1], namely Smoothed Multi Crack Concrete (model 102). The specifications of SR EN 1992-1-1/2004 [6] and SR EN 1993-2:2007 [7] were used for the elastic characteristics of the two materials. 
The beam was first loaded with the permanent weight, in order to establish the height to which the cross-section develops cracks. The analysis suggested that the cracks appear around $80 \%$ of the total loading. The cracks for $100 \%$ of the total loading are shown in Figures 6 and 7.

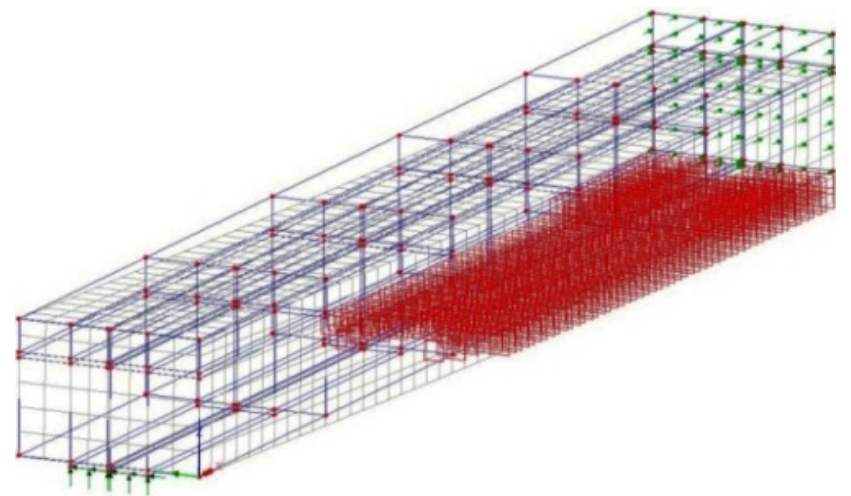

Fig. 6 - The cracks for $100 \%$ of the total loading with the permanent weight (3D)
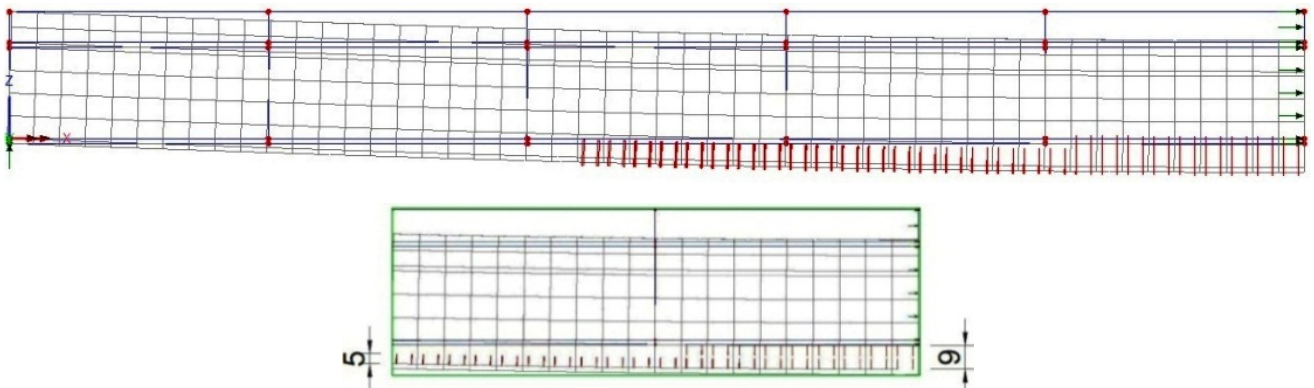

Fig. 7 - The cracks for $100 \%$ of the total loading with the permanent weight (2D)

As can be seen in Figure 7, the crack length from the permanent weight on the mid-span of the bridge is about $9 \mathrm{~cm}$.

\section{Loading with traffic actions (LM71 model)}

Subsequently, the beam with reduced section (cracked concrete from the permanent weight removed) was loaded with traffic actions. The cracked concrete was removed stepwise to obtain a regular mesh. The beam with reduced cross-section is shown in Figure 8.

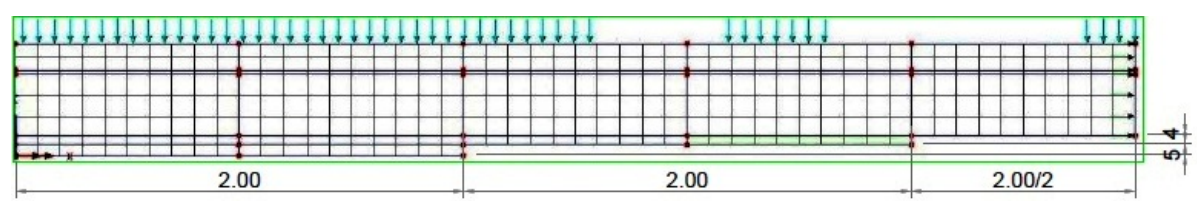

Fig. 8 - The beam with reduced cross-section from the permanent weight

The calculation model contains 3530 "3D" finite elements type "Stress" HX8M and 4696 nodes, resulting in 14088 degrees of freedom, as shown in Figure 9.

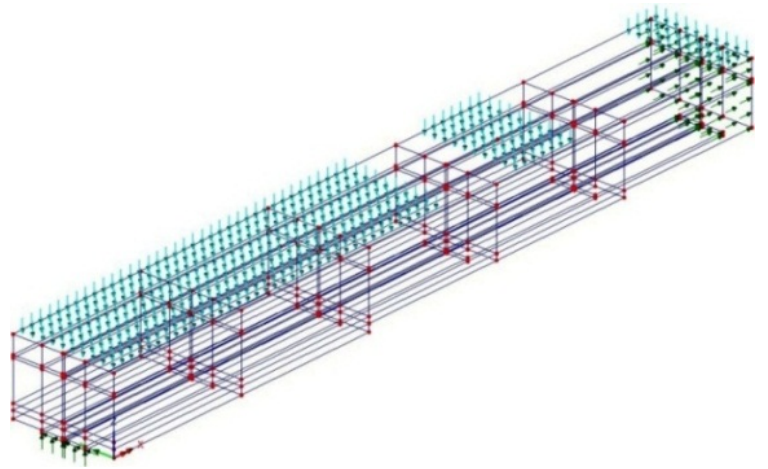

Fig. 9 - The calculation model for traffic actions (LM71 model) 
The LM71 model was applied in fixed position, of maximum. The analysis suggests that the cracks appear around $40 \%$ of the total loading with the LM71 model. The Figures 10 and 11 present the cracks for $100 \%$ of the loading with the LM71 model.

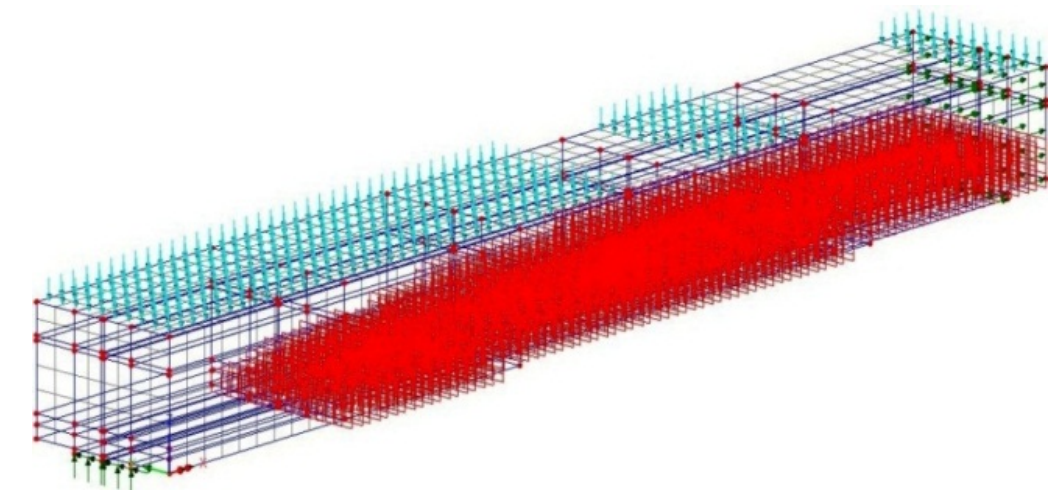

Fig. 10 - The cracks in concrete $-100 \%$ of the load with the LM71 model (3D)
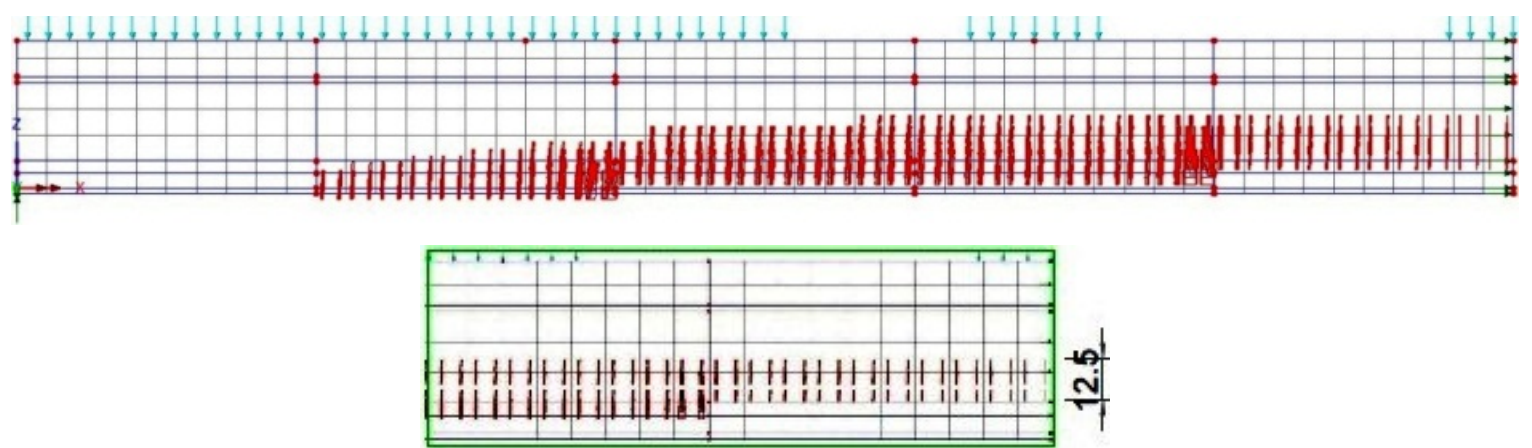

Fig. 11 - The cracks in concrete $-100 \%$ of the load with the LM71 model (2D)

As can be seen in Figure 11, the crack length on the mid-span of the bridge is about $12,5 \mathrm{~cm}$.

For further calculations, the cracked concrete from both permanent weight and traffic actions was removed. The outcome was a beam with reduced cross-section, which will be used in subsequent analyzes, as shown in Figure 12.

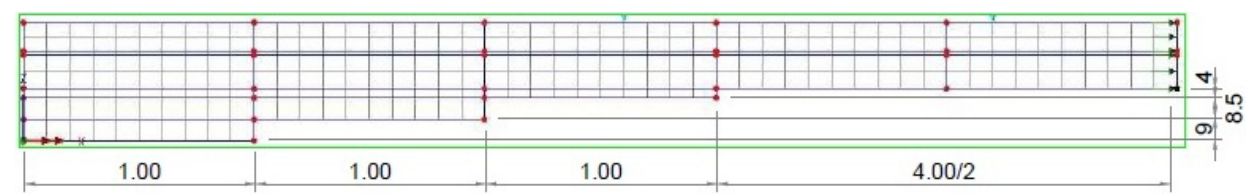

Fig. 12 - The beam with reduced cross-section from the permanent weight and traffic actions $(\mathbf{L}=\mathbf{1 0 m})$

\subsubsection{Bridge with the $20 \mathrm{~m}$ opening}

The analysis presented at the $10 \mathrm{~m}$ opening was resumed, and thus, it was obtained the beam with reduced cross-section which will be used in subsequent analyzes, as shown in Figure13.

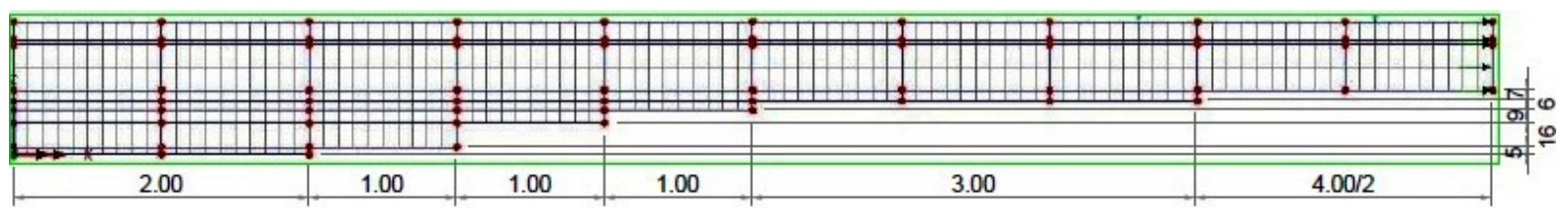

Fig. 13 - The beam with reduced cross-section from the permanent weight and traffic actions $(\mathbf{L}=\mathbf{2 0 m})$

\subsubsection{Bride with a 30 m opening}

The analysis presented at the $10 \mathrm{~m}$ opening was resumed, and thus, it was obtained the beam with reduced cross-section which will be used in subsequent analyzes, as shown in Figure14. 


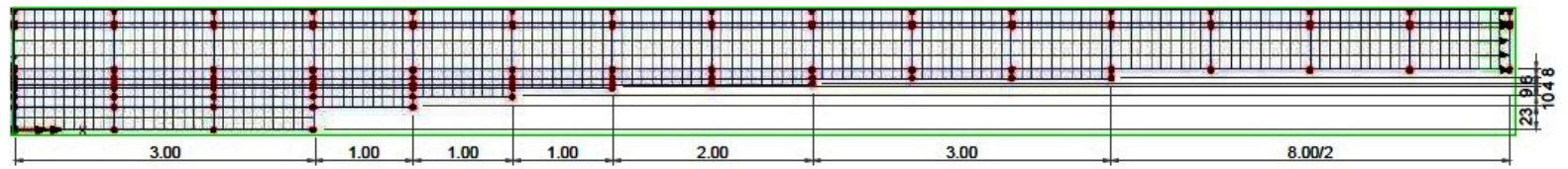

Fig. 14 - The beam with reduced cross-section from permanent weight and traffic actions $(\mathbf{L}=\mathbf{3 0 m})$

\subsection{Presentation of the calculation models}

\section{Bridge with $L=10 m$ opening}

The calculation model contains 18780 "3D” finite elements type "Stress" HX8M and 24171 nodes, resulting in 72513 degrees of freedom, as shown in Figure 15.
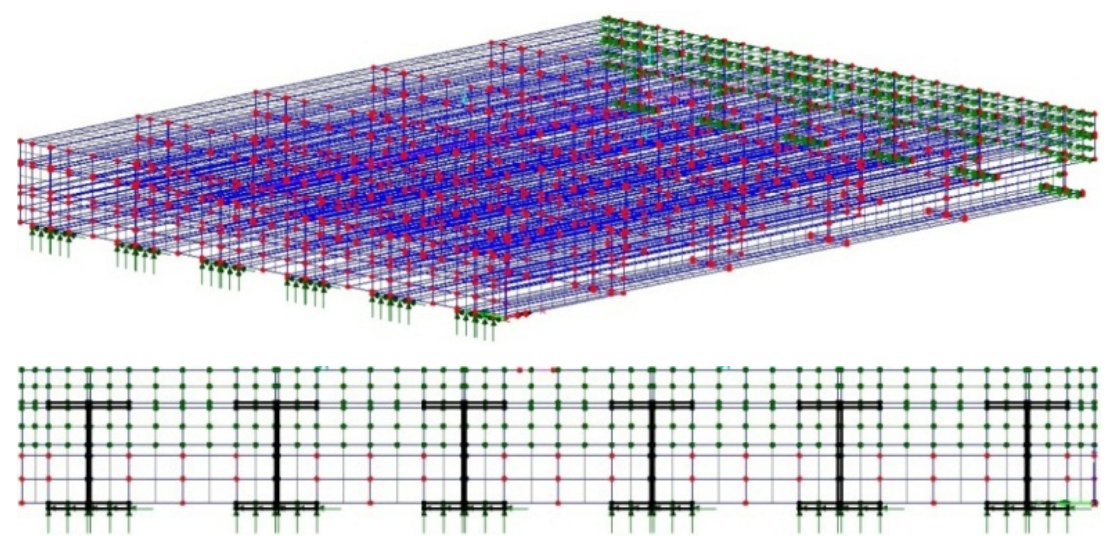

Fig. 15 - The calculation model and the cross-section of the bridge with $\mathbf{L}=\mathbf{1 0 m}$ opening

\section{Bridge with $L=20 m$ opening}

The calculation model contains 22080 "3D” finite elements type "Stress" HX8M and 29374 nodes, resulting in 88122 degrees of freedom, as shown in Figure 16.
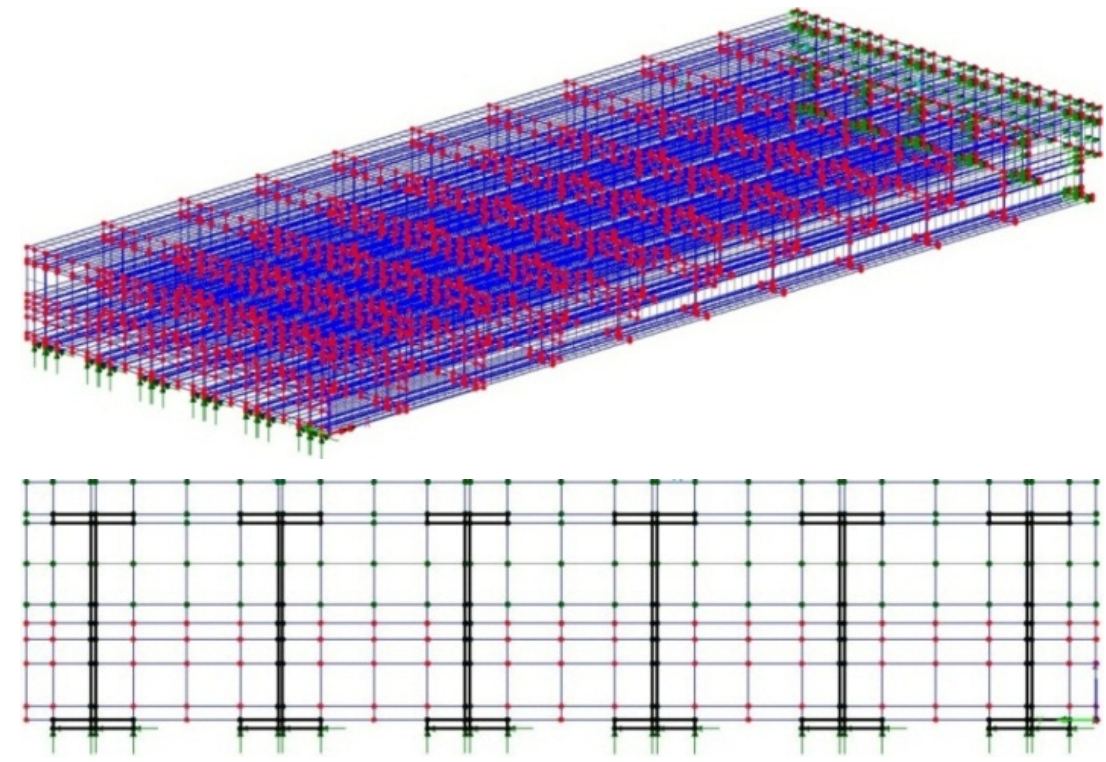

Fig. 16 - The calculation model and the cross-section of the bridge with $\mathbf{L}=\mathbf{2 0 m}$ opening

\section{Bridge with $L=30 m$ opening}

The calculation model contains 41100 “3D” finite elements type "Stress" HX8M and 53567 nodes, resulting in 160701 degrees of freedom, as shown in Figure 17. 

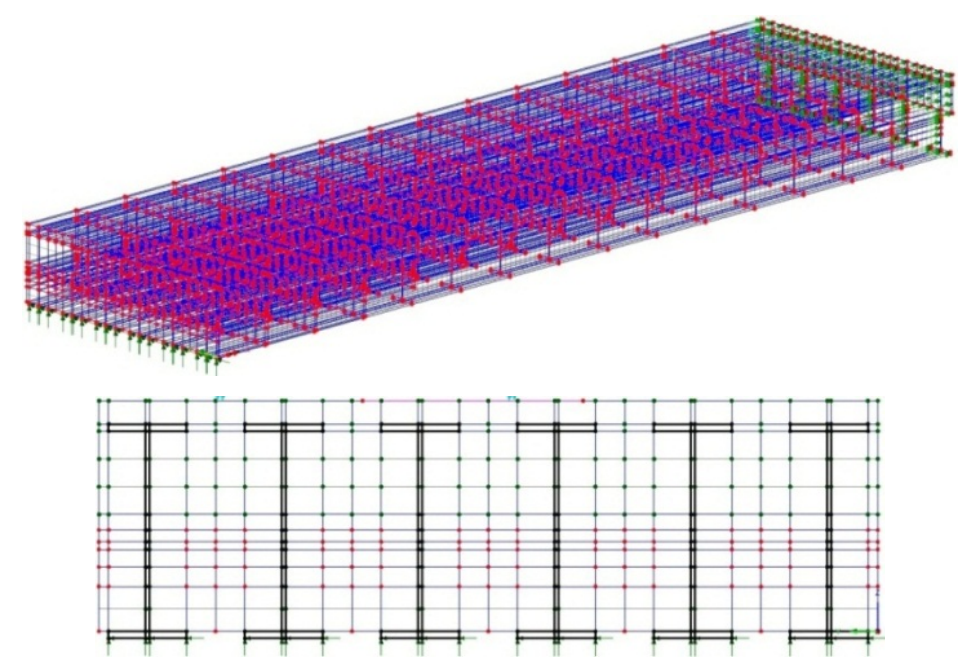

Fig. 17 - The calculation model and the cross-section of the bridge with $\mathbf{L}=\mathbf{3 0 m}$ opening

\subsection{Calculation of the forces in curve}

Within the analysis, only the traffic loads and the centrifugal force were taken into consideration. The specifications of SR EN 1991-2/2005 [5] were used for their calculation. The forces in curve are shown in Figure 3 and represent:

- $\quad Q_{t k}$ - the direct effect of the centrifugal force with its characteristic value;

- $\quad Q_{t k}^{\prime}$ - the indirect effect of the centrifugal force with its characteristic value;

- $Q_{v k}$ - the characteristic value of the vertical load of the LM71 model.

\subsection{The highlight of results}

For each analyzed case, the values of the bending moments in the middle section of each longitudinal beam were computed using the FEM Lusas program [1]. These are presented summarized in Table 2.

Bending moments from FEM analysis

Table 2

\begin{tabular}{|c|c|c|c|c|c|c|c|}
\hline \multirow{2}{*}{ 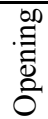 } & \multirow{2}{*}{ Radius } & \multicolumn{6}{|c|}{ Bending moments (kNm) } \\
\hline & & Beam 1 & Beam 2 & Beam 3 & Beam 4 & Beam 5 & Beam 6 \\
\hline \multirow{6}{*}{ 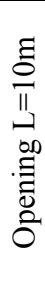 } & $\mathrm{R}=100 \mathrm{~m}$ & 343.6 & 338.6 & 318.5 & 305.7 & 291.6 & 268.6 \\
\hline & $\mathrm{R}=400 \mathrm{~m}$ & 350.6 & 343.8 & 319.7 & 304.2 & 286.7 & 261.2 \\
\hline & $\mathrm{R}=700 \mathrm{~m}$ & 351.5 & 344.5 & 319.9 & 304 & 286 & 260.1 \\
\hline & $\mathrm{R}=1000 \mathrm{~m}$ & 348.5 & 342.8 & 319.4 & 304.7 & 287.9 & 263 \\
\hline & $\mathrm{R}=1500 \mathrm{~m}$ & 344.3 & 340.2 & 318.6 & 305.7 & 290.5 & 266.9 \\
\hline & without curve & 318.4 & 324.6 & 313.7 & 311.4 & 306.6 & 291.4 \\
\hline \multirow{6}{*}{ 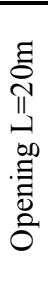 } & $\mathrm{R}=100 \mathrm{~m}$ & 1160 & 1153 & 1109 & 1071 & 1036 & 959.5 \\
\hline & $\mathrm{R}=400 \mathrm{~m}$ & 1158 & 1157 & 1108 & 1071 & 1034 & 956.1 \\
\hline & $\mathrm{R}=700 \mathrm{~m}$ & 1158 & 1158 & 1109 & 1071 & 1034 & 955.6 \\
\hline & $\mathrm{R}=1000 \mathrm{~m}$ & 1150 & 1153 & 1107 & 1073 & 1039 & 963.3 \\
\hline & $\mathrm{R}=1500 \mathrm{~m}$ & 1139 & 1147 & 1105 & 1075 & 1045 & 973.4 \\
\hline & without curve & 1088 & 1116 & 1096 & 1086 & 1077 & 1023 \\
\hline \multirow{6}{*}{ 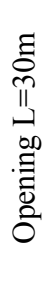 } & $\mathrm{R}=100 \mathrm{~m}$ & 2404 & 2356 & 2267 & 2182 & 2104 & 1979 \\
\hline & $\mathrm{R}=400 \mathrm{~m}$ & 2367 & 2342 & 2259 & 2187 & 2118 & 1999 \\
\hline & $\mathrm{R}=700 \mathrm{~m}$ & 2362 & 2340 & 2258 & 2188 & 2120 & 2002 \\
\hline & $\mathrm{R}=1000 \mathrm{~m}$ & 2345 & 2330 & 2255 & 2191 & 2130 & 2018 \\
\hline & $\mathrm{R}=1500 \mathrm{~m}$ & 2324 & 2317 & 2251 & 2196 & 2143 & 2038 \\
\hline & without curve & 2233 & 2263 & 2233 & 2215 & 2198 & 2126 \\
\hline
\end{tabular}


5. Variation charts of the bending moments depending on radius curve of the track axis

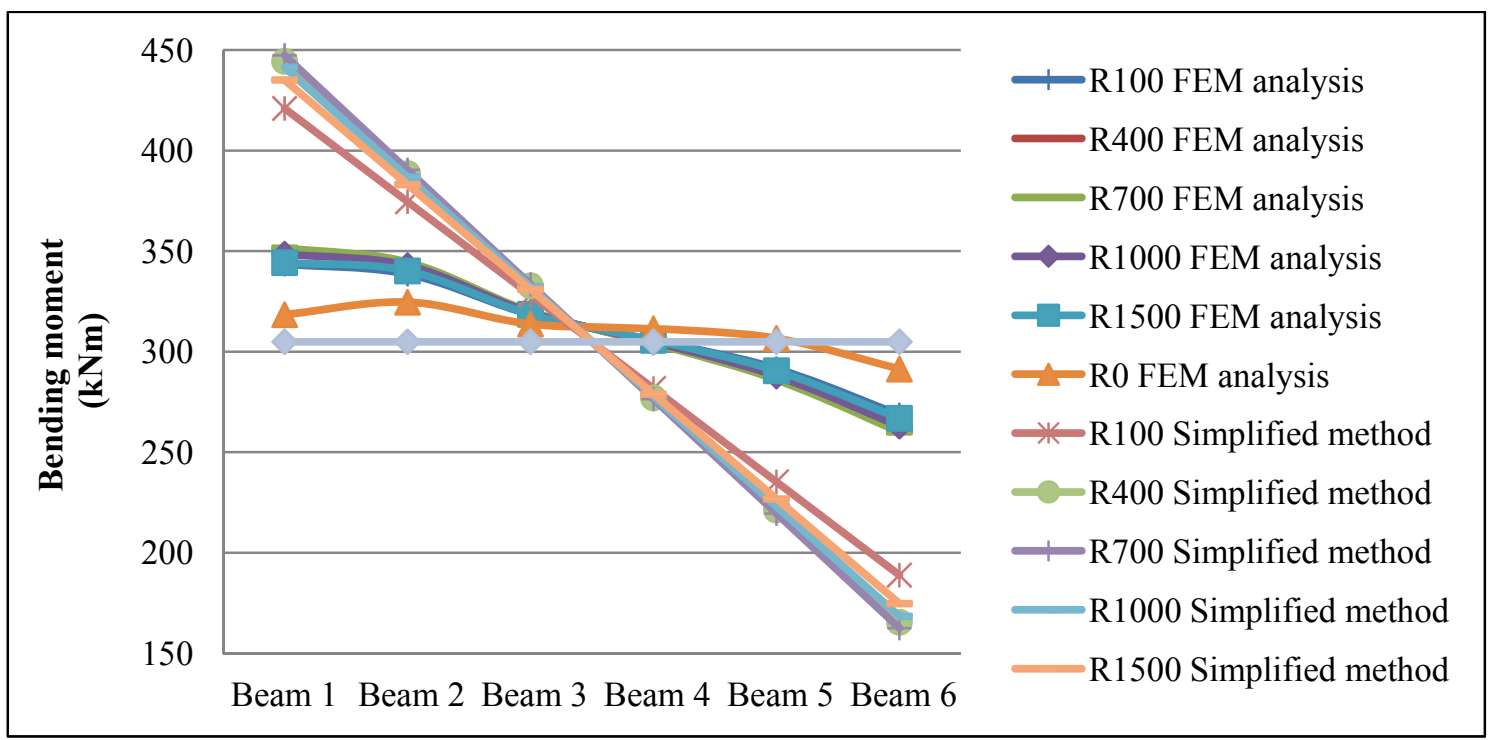

Fig. 18 - Transversal variation of the bending moment produced by LM71 action, for the section at midspan of the bridge with $\mathrm{L}=10 \mathrm{~m}$ opening, depending on the radius

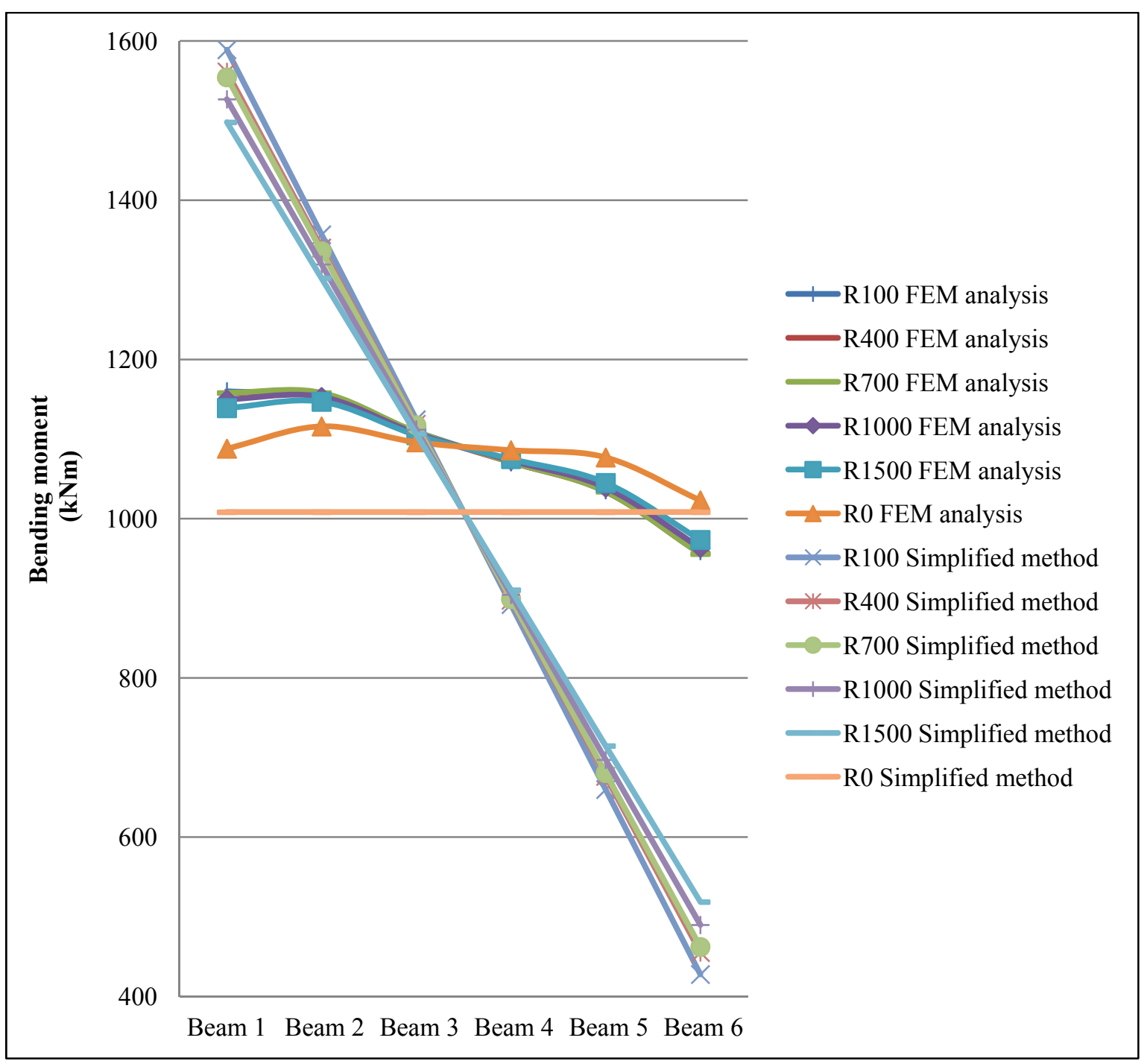

Fig. 19 - Transversal variation of the bending moment produced by LM71 action, for the section at midspan of the bridge with $\mathrm{L}=20 \mathrm{~m}$ opening, depending on the radius 


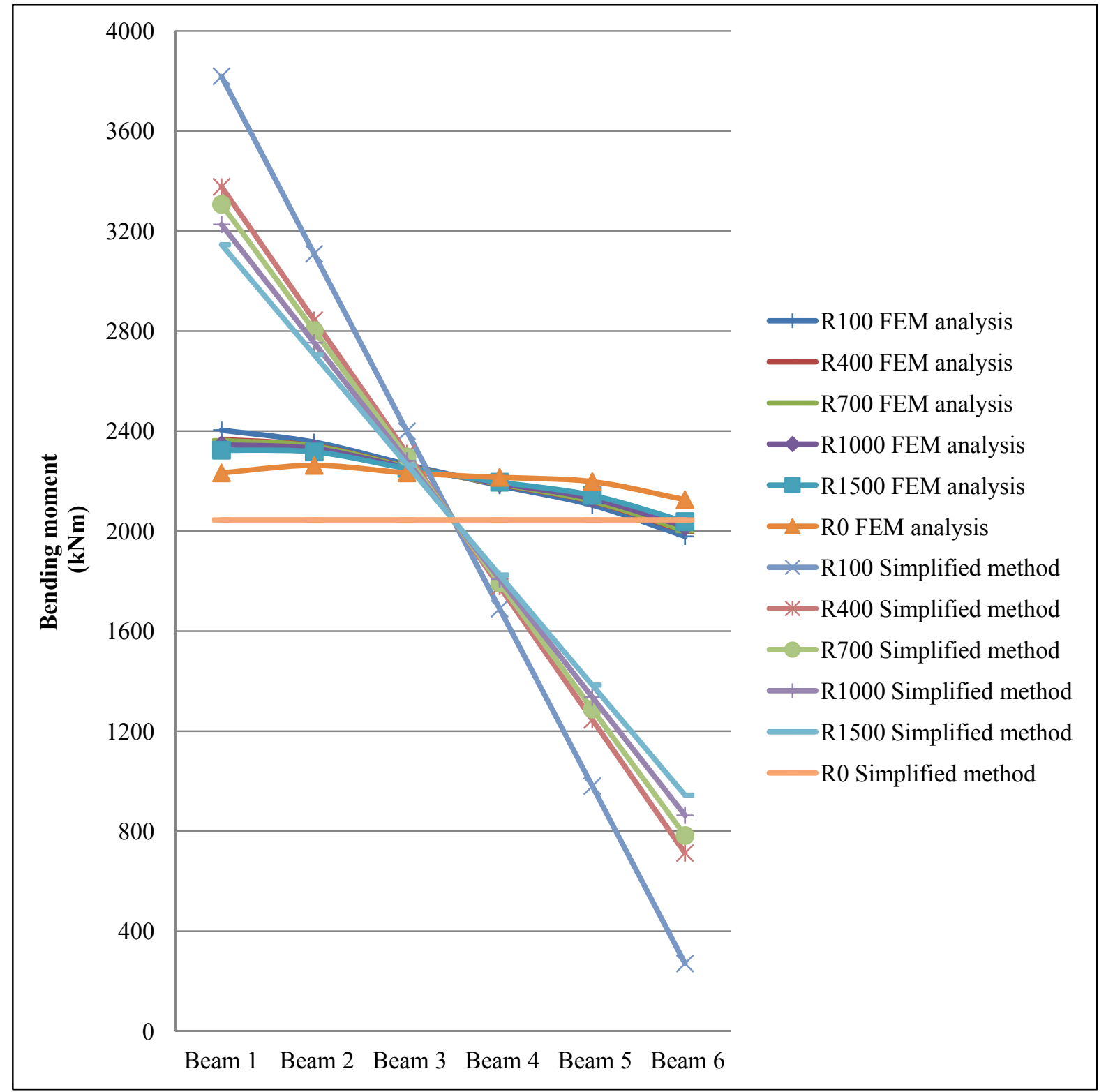

Fig. 20 - Transversal variation of the bending moment produced by LM71 action, for the section at mid-span of the bridge with $\mathrm{L}=30 \mathrm{~m}$ opening, depending on the radius

\section{Conclusions regarding the influence of the curvature of the track axis}

When it comes to bridges with the track axis in curve, it can be noted an overload of the beams from outside of the curve and an underload of the beams from inside of the curve, in relation with the situation of the bridge with the track axis in alignment, as shown in Figures 18 up to 20. Practically, with respect to bridges with the track axis in curve, the variation graphs of the bending moments are obtained by rotating around the bridge deck axis of the variation graphs of the bending moments for bridges with track axis in alignment.

Also, it is noted that at small openings the greatest efforts appear for radius $\mathrm{R}=700 \mathrm{~m}$, for which the centrifugal force has the highest values from all the analyzed radii. As the openings increase the maximum values are obtained for the radius $\mathrm{R}=100 \mathrm{~m}$, because the forces depart significantly from the bridge deck axis.

Another finding shows that the bending moments from the traffic actions have approximately the same values for the analyzed radii $\mathrm{R}=100 \mathrm{~m}-1500 \mathrm{~m}$ (see Figures 18 up to 20), for all the three analyzed openings. 
In addition, the graphics from the $10 \mathrm{~m}$ opening is slightly different in comparison with the graphics from the 20 and $30 \mathrm{~m}$ openings. The difference can be observed at the $10 \mathrm{~m}$ opening, namely the deck is closer to the case of the plates with equal dimensions where the bending is spherical while for the other openings, where the contrast between the two dimensions is much bigger ( $4 \mathrm{~m}$ and $20 \mathrm{~m} / 4 \mathrm{~m}$ and $30 \mathrm{~m}$ as opposed to $4 \mathrm{~m}$ and $10 \mathrm{~m}$ ), the bending is cylindrical.

When it comes to the simplified method, an overstatement of the bending moments from the traffic actions due to the track axis curvature can be noted, towards the situation in alignment. Thus, at the $10 \mathrm{~m}$ opening, the findings show an increase of $46.7 \%$ of the maximum bending moment , at the $20 \mathrm{~m}$ opening an increase of $57.6 \%$, respectively at the $30 \mathrm{~m}$ opening an increase of $86.7 \%$ (as shown in Figure 21). In case of the FEM analysis, the maximum increase of the bending moment due to the track axis curvature was $8.3 \%$.

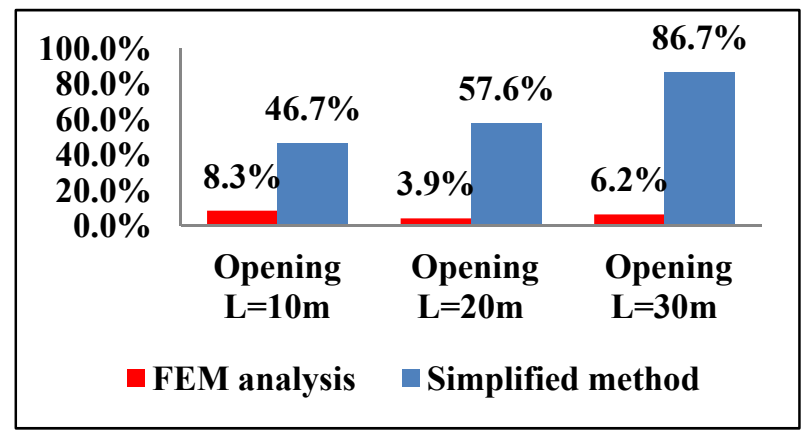

Fig. 21 - The increase in the bending moment produced by the LM71action due to the presence of the curve towards the situation of the bridge the with track axis in alignment

Moreover, the differences between the maximum bending moment determined by the simplified calculation and those determined by the FEM analysis become very important as the opening increases. At the $10 \mathrm{~m}$ opening, the difference between the two calculations is $27.3 \%$, at the $20 \mathrm{~m}$ opening the difference is $37 \%$ and at the $30 \mathrm{~m}$ opening the difference is $58.9 \%$ (as shown in Figure 22).

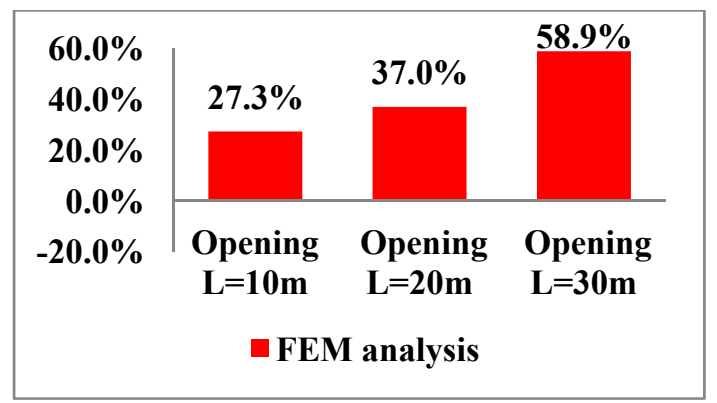

Fig. 22 - The difference between the maximum bending moment produced by LM71 obtained by FEM analysis towards those calculated with simplified method

The overall conclusion regarding the influence of the track axis curvature at railway filler-beam deck bridges is that the stress state obtained by the simplified method calculation is significantly overstated. Therefore, when it comes to bridges with the track axis in curve it is recommended to perform calculation using finite element programs.

\section{References}

[1] http://www.lusas.com

[2] UIC Code 773-4 R. (1997). International Union of Railways, F-75015. Paris

[3] SR 1911-98. (1998). Poduri metalice de cale ferată - Prescripţii de proiectare.

[4] Design Tables for Filler Beam Railway Bridges. (1999). International Union of Railways, 16, rue Jean Rey, F-75015. Paris.

[5] SR EN 1991-2:2005. (2005). Eurocode 1: Acţiuni asupra structurilor, Partea 2: Acţiuni din trafic la poduri.

[6] SR EN 1992-1-1/2004. (2006). Proiectarea structurilor de beton. Reguli generale şi reguli pentru clădiri.

[7] SR EN 1993-2:2007. (2007). Proiectarea structurilor de oțel. Poduri de oțel. 